\title{
The construction of scientific and technological service platform in industrial clusters
}

\author{
ZHANG Linlin $^{1, a}$, ZHAO Haiming ${ }^{1,2, ~ *}$, LIAO Xiaole ${ }^{1, b}$, Ji Yaqian $^{1, c}$ \\ ${ }^{1}$ College of Mechanical and Electrical Engineering, Central South University, Changsha, 410083, \\ China \\ ${ }^{2}$ State Key Laboratory of High Performance Complex Manufacturing, Central South University, \\ Changsha, 410083, China \\ aemail: 984120969@qq.com, Corresponding author*email:

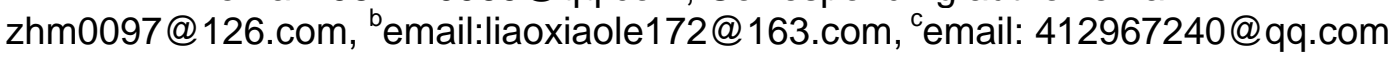

Keywords: Engineering machinery industrial cluster; Scientific and technological service platform; Functional module division

\begin{abstract}
The collaborative development of each unit in industrial clusters is the only way to enhance the regional industrial competitiveness and the regional economy. While the scientific and technological service platform based on integrated source information is an important carrier for enterprise cluster to achieve collaborative development. In this paper, by analyzing the development condition of Hunan Engineering Machinery Industrial Cluster, the service needs are proposed and different function modules of the resources in industrial clusters are divided in order to satisfy the service requirements. In addition, the operation process program to the network operation of the scientific and technological service platform.
\end{abstract}

\section{Introduction}

With the development of globalization, collaborative development is the only way for the survival and development of modern enterprise at present. The idea of development of industrial clusters is finding the competitive advantage of specific regional starting from the whole. The idea breaks through the boundaries of the enterprise and the single industry and focuses on the interactions of the competitive and cooperative enterprises, relevant institutions, government and non-governmental organizations in particular areas. This can make the harmonious development of economy and society be considered systematicly from the whole region rather than considering the interests of some individuals or some narrow regions. Besides, the industry clusters that can be a specific regional competitive advantage are also investigated and the competition and cooperation of adjacent areas are considered. With the informationalized service management mode, the resources superiority and service needs of enterprises can be delivered to each enterprise in industry cluster by networked information service platform. This can build a bridge for enterprise cooperation and promote the application and development of the industry-university-research cooperation in industry cluster. This type of science and technology service platform has been widely applied in many industry clusters in China, which promotes the whole development of industry cluster to a great extent.

The core enterprises of Hunan Engineering Machinery Industrial Cluster include some large-scale enterprises such as ZOOMLION, Sany and Sunward Intelligent, as well as some small and medium enterprises such as Changsha party pneumatic hydraulic electromechanical Co. Ltd, Changsha hydraulic parts factory and Zhuzhou kuohai hydraulic pneumatic complete equipment company. Hence it has obvious cluster characteristics. Throughout the development situation of each enterprise, since the scientific and technological service platform for the cluster units was fail to be established, there are enough competition but inadequate cooperation between enterprises. Hence the core enterprise in this region is high production costs, while the small and medium enterprises development is slow and the transfer ratio of research achievement is low $[1,2]$. 


\section{Function module division of the service information platform}

The construction of the information platform must depend on the development condition of the industry cluster and meet the development needs. The application of modular design theory to the construction program of scientific and technological innovation service platform can greatly improve the service quality and efficiency, which can effectively complete the integration of various resources only when. The science and technology innovation service in the industrial cluster can be divided into several service modules: the basic equipment service module, the collaborative product development service module, the high technology and advanced applicable technology transfer service module, the intellectual property, the electronic commerce and finance service module and the industrial design service module. All above can effectively solve the problems faced by many small and medium enterprise and provide information services such as enterprise establishing, technology research and development, promotion of achievements, technology transaction, risk investment, financing guarantee, exchange of personnel, management consulting, information dissemination and so on for the comprehensive development of cluster. The following is the contents provided by the service module:

(1)The basic equipment service module: providing information message of relevant laboratory, detection mechanism and other infrastructure information in industry cluster. Besides, it provides integrated information consultation and inquiry service for industry experiment, collection of testing resources, sharing and exchange of information, knowledge retrieval, experiments and detection application service system. Since universities and research institutes have advanced experiment system while core enterprises and their supporting enterprises can provide detecting equipment of specific application, integration of the information resources can greatly shorten the product development cycle.

(2)The collaborative product development service module: the module integrates the technical force, personnel resources, product information, documents, standards and other information of participating units in industry cluster. And it can provide a series of unified collaborative R \& D internal standards and a platform of research communication and knowledge sharing. This can achieve a seamless docking of the technique requirements of each unite. A unified centralized-distributed PDM architecture is constructed which can be effectively and stably applied by internal staff of cluster. A unified collaborative tool is used and a unified exchange platform is used for the interaction of written communication, language communication and network conference/remote collaboration.

(3)The transfer service module of new technology and advanced applicable technology: the module mainly integrates information such as the advantaged industrial resources, overseas science and technology resources, technology and talent demand, invite investment, investment and financing, project packaging, scientific and technological achievements, patents, standards, experts, research institutions, basic science and technology condition, intermediaries, expert consultation, exhibition information, management, industry dynamics. It can provide technical and economic information services for all kinds of innovative subject. [5]

(4)The intellectual property, electronic commerce and finance service module: the module integrates intellectual property and financing, loans, guarantees and other information resources for small and medium enterprise. It provides a one-stop innovation service platform of resource-sharing, information-exchange and intelligent search. There are many excellent coordination institutions of law and finance in industry cluster. These institutions has specialized talent team which can provide reasonable suggestions and supports to intellectual property and financial policy issues.

(5)The industrial design service module: this module is mainly for the construction of industrial design information system. The databases of engineering machinery products, industrial design expert and knowledge are established. The public industrial design technology supports the system construction and provides industrial design technology support for engineering machinery enterprises. Besides, it also includes the construction of the industrial design technique promotion and the personnel training system.

The networking science and technology service platform can integrate all the above service 
modules. In this way, users can seek consulting and management of relevant business in corresponding service module. This can achieve the seamless docking of each service conveniently.

\section{Applicable customer of science and technology service platform}

The construction of an enterprise cluster is always complex. It involves not only enterprises, institutions but local universities and scientific research institute. The enterprises involve leading enterprises and its corresponding supporting enterprises. The institutions include functional government departments and media service department. As a national pilot reform, Hunan engineering machinery industrial cluster which takes Changsha Zhuzhou Xiangtan cluster as the main body has already been one of the most important base of engineering machinery manufacturing industry in china. The scientific and technological innovation and industrialization promotion in cluster require mutual cooperation. Hence the science and technology service platform should attract institution constructions of every section including developing, manufacturing and distributing to form a complete service system.

As for the Hunan engineering machinery industrial cluster, the information service platform can attract the following organizations:

(1) Developing and manufacturing enterprises: with ZOOMLION, Sany and Xiangtan motor and other large-scale enterprises as the core and several parts supporting enterprise as the auxiliary, Hunan province has achieved the "south pole" in "pole" of China engineering machinery field. Thereinto, Changsha has more than 30 large-scale engineering machinery enterprises and they mainly produce more than 400 specifications of products which can be classified into 12 major categories and more than 100 sub-categories. Now Changsha has become the highest concentration of engineering machinery in our country.[6,7]

(2) Research institutes and Local Universities: the industrial cluster has gathered a large number of key universities and scientific research institutions, including Central South University, Hunan University, National University of Defense Technology, Xiangtan University, Hunan University of Science and Technology, Hunan Institute of Engineering, Changsha Research Institute Of Mining And Metallurgy, Changsha Institute of mining research, etc. These research institutes and universities can provide a number of technical support and personnel training for the development of the engineering machinery industry cluster and the motor industry cluster.

(3) Logistics service unit: according to the statistics of Hunan Provincial Federation of logistics and purchasing, there are more than 50 thousand logistics economic organizations until the end of 2013. Among them, more than 3100 logistics enterprises have qualifications of a legal person. As of July 2012, 88 logistics enterprises in Hunan have been recognized by countries as class A, 69 of which are in Changsha, Zhuzhou and Xiangtan.

(4) Government sector: the development of industrial clusters cannot do without the support of government policy. Relevant government departments mainly are Hunan science and Technology Department, Commerce Department of Hunan Province, CCPIT etc.

(5) Financial department: since the financial institutions can provide the capital chain needed by the industrial cluster, it is regarded as the blood of economic development. At present, the relevant financial institutions in Hunan province are Hunan branch of China bank, Industrial and Commercial Bank of China, the Agricultural Bank of China and China Construction Bank.

(6) Law firm: there are many well-known law firms in Hunan province, such as Hunan Tiandiren law firm, Hunan Tongcheng law firm, Hunan Jinzhou law firm, Jiaoyang law firm of Hunan China etc. These firms can provide market management affairs that may be produced during the development of industrial clusters such as intellectual property, investment and financing, legal counsel, financial and securities and company management.

(7) Entrepreneurial intermediary service institutions: at present, there are many intermediary service institutions with a certain scale in Hunan such as productivity promotion center, business service center, engineering technology research center etc. According to the statistics of National Productivity Promotion Center, there are 43 Productivity Promotion Centers in Hunan until the end 
of 2012.

\section{Networking operation of the science and technology platform}

The science and technology service platform is the carrier to realize innovation service mode of the industry clusters. By modular division to the service resources, the service information may be with strong pertinence. The science and technology service platform can be divided into 5 modules: public information, user management, dynamic issue of service module, friendly links and advertisement. Through the five modules, publics and members can clearly search their information.

(1) Public information: it involves a brief introduction of its function and the help of operation.

(2) User management: it is built mainly for the administrator and members of the platform. Since the administrators and members can share more services than publics, it is necessary to provide specific authority management foe them. It mainly includes "User Login", "User Registration", "Crypto service", "Online advisory services "and "BBS of members".

(3) Dynamic issue of service module: it's a section needing to update timely, and the content mainly involves the resource information of the function module. It will publish the service demand of each unit, new product information and new policy issued information to members of platform and the public timely.

(4) Friendly links and advertisement: the friendly links is not the application part of the platform. It is used for the quick connection of related units when needed, which can effectively share resources of each unit and enhance mutual communication. Objects involved in friendly links are representative leading enterprises, relevant legal department, government departments, universities, research institutes and financial institutions in or out of the industry cluster. The advertisement layer is constructed aimed at the profit itself and it don't participate in the innovation service activities directly. Meanwhile, it can also promote the rapid development of small and medium enterprise.

The networking operation of the science and technology service platform starts at the platform gateway and then enters into different roles after the authentication of user management (administrators or members). Then users can enter into dynamic issue of service module. According to different service needs, they can enter into the corresponding service module system and select the needed service information. Meanwhile, they can give feedback such as service intent and advice to users to communicate through web forum. The operation process is shown as follows: 


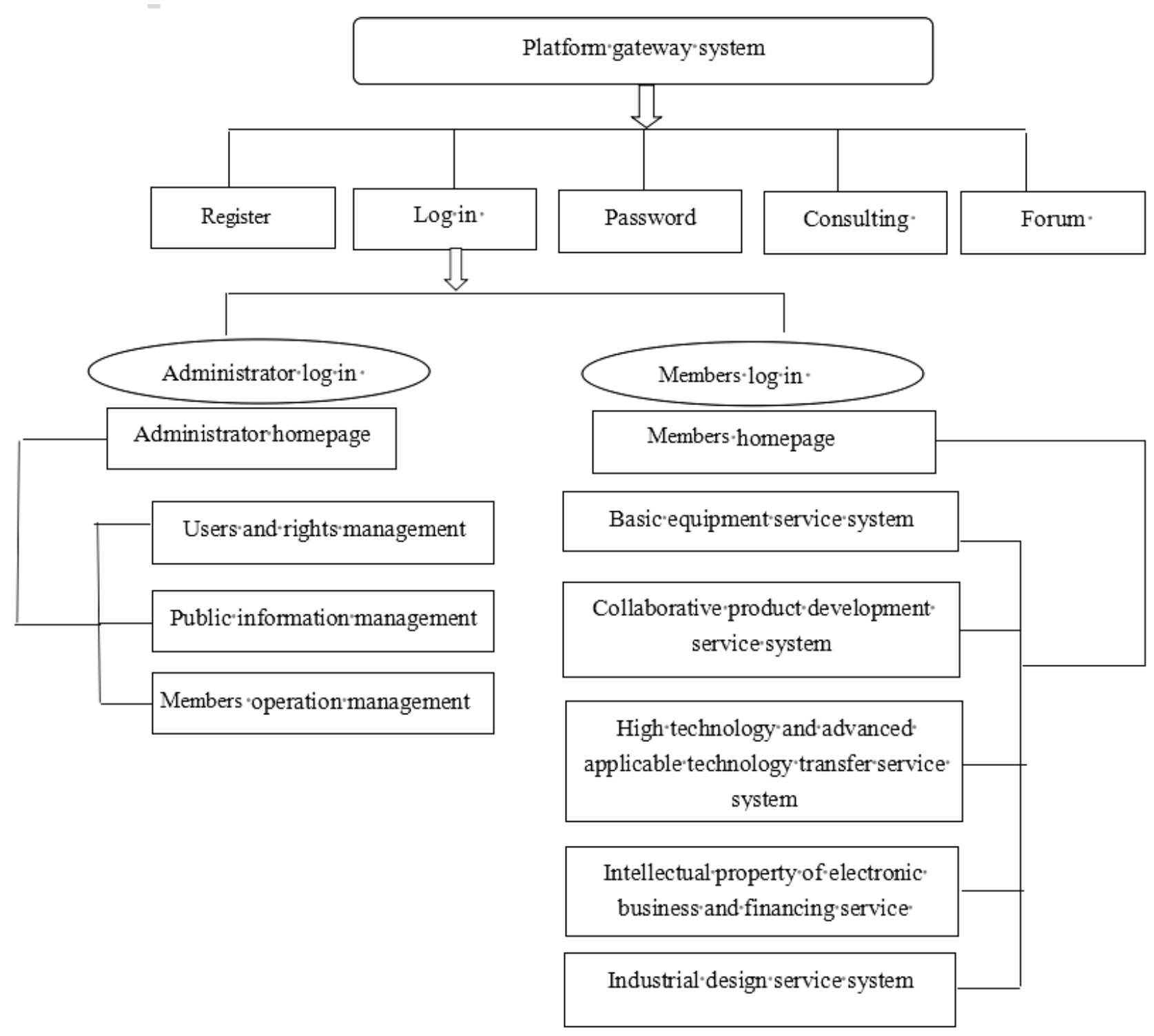

Fig.1 Operation process of networking platform

\section{Summary}

With the acceleration of product updating and continuous progress of information technology, the collaborative development pattern in industry cluster will be adapted by each enterprise. The science and technology service platform will also continue to play its role. In this paper, the construction of the science and technology service platform in Hunan engineering machinery industry cluster is studied. It mainly completes the following works:

(1) The development condition of industry cluster is analyzed.

(2) Service needs are proposed according to industrial characteristics in this region.

(3) Different function service modules are established corresponding to service needs.

(4) The networking operation program of platform is put forward.

The platform can clearly show all resources and demands in industry cluster to members and closely contact each unit. This can form a rapid information flow and improve the collaborative development of the industry cluster. The small and medium enterprises can be developed rapidly under the collaborative development environment, which can reduce the production cost of core enterprises. At the same time, the conversion rate of scientific research achievements can be improved with the help of the science and technology innovation of universities and research institutes. 


\section{Acknowledgements}

This work was supported by the national science and technology support plan industry cluster technology innovation service platform under the support of common key technology research and development 2012BAH11F01, China.

\section{References}

[1] YANG Shui-gen. Research of Hu-nan Engineering and Construction Mechanic Industrial Cluster's Based On Industrial Chain. Journal of hunan business college, 2007, 14(5):47-49.

[2] Liu Ling, Tao Xiao-ai, Xiong Yu. Changsha cast "China construction machinery of all" [N], Hunan Daily, 2007-08-29(A01).

[3] LI Guo-xi, WU Jian-zhong, ZHANG Meng. Approach to Product Modular Design Based on FPBS[J]. Journal of national university of defense technology, 2009, 31(5): 75-80.

[4] Ho H, Haung C, Yang H. Development of Modular Services[C].IEEE Computer Society, Washington,2009.

[5] Tan Gang. Basic Ideas of Introducing Innovative Scientific and Technological Service Platform for SMEs in Guangxi [J]. Enterprise Science and Technology \& Development, 2010,12 :1-3.

[6] HOU Jin-song, PENG Yue-xiang. Strategy Research of Development of Hunan Engineering Machinery Industry[J]. JOURNAL OF HUNAN INDUSTRY POLYTECHNIC, 2007, 7(4):28-30.

[7] Yang Yan-fang. The Development Research On Electromechanical Industrial Agglomeration of ChangZhuTan City Group[D]. China University of Geosciences (Wu Han), 2008. 